\title{
MEDIDAS FACIAIS ANTROPOMÉTRICAS DE ADULTOS SEGUNDO TIPO FACIAL E SEXO
}

\author{
Adult facial anthropometric measurements according \\ to facial type and gender
}

\author{
Rossana Ribeiro Ramires (1), Léslie Piccolotto Ferreira (2), Irene Queiroz Marchesan ${ }^{(3)}$, \\ Débora Martins Cattoni ${ }^{(4)}$, Marta Assumpção de Andrada e Silva ${ }^{(5)}$
}

\begin{abstract}
RESUMO
Objetivo: descrever medidas antropométricas faciais de adultos, segundo tipo facial e sexo. Método: participaram 105 adultos leucodermas, 34 (32,4\%) homens e $71(67,6 \%)$ mulheres, com idades entre 20 e 40 anos, de uma clínica particular de ortodontia de Belo Horizonte, Minas Gerais. Foram comparados os achados da análise cefalométrica para determinação do tipo de face com sete medidas antropométricas faciais diretas: altura facial anterior- násio ao mentoniano; distância bizigomáticazigomático esquerdo ao direito; altura do terço facial inferior- subnasal ao mentoniano; altura da face média- násio ao estômio; altura do queixo- supramentoniano ao mentoniano; altura da face inferiorestômio ao mentoniano; e altura facial posterior- condílio ao gônio. Resultados: para o sexo masculino, os tipos faciais classificados por meio da cefalometria apresentaram diferenças estatisticamente significantes para os valores médios das medidas antropométricas: altura facial anterior, altura do terço facial inferior, altura da face média e altura da face inferior. Para o sexo feminino, diferenças estatisticamente significantes foram encontradas nas medidas: altura facial anterior, altura do terço facial inferior, altura da face média, altura da face inferior e altura facial posterior. Conclusão: houve dimorfismo sexual para todas as medidas antropométricas obtidas, as quais tiveram valores médios maiores para o sexo masculino quando comparados ao feminino. Os tipos faciais classificados por meio da cefalometria apresentaram diferenças estatisticamente significantes para quatro medidas antropométricas do sexo masculino e cinco do feminino.
\end{abstract}

DESCRITORES: Face; Medidas; Antropometria; Adulto

(1) Fonoaudióloga; Integrante do Corpo Técnico Diretor do Grupo de Apoio ao Indivíduo com Autismo- GAIA de São José dos Campos, São Paulo; Mestre em Fonoaudiologia pela Pontifícia Universidade Católica de São Paulo.

(2) Fonoaudióloga; Professora Titular da Graduação e PósGraduação em Fonoaudiologia da Pontifícia Universidade Católica de São Paulo, PUCSP, São Paulo, SP; Coordenadora e docente do Curso de Especialização em Fonoaudiologia - Voz da Coordenadoria Geral de Especialização, Aperfeiçoamento e Extensão da Pontifícia Universidade Católica de São Paulo, COGEAE-PUC/SP, São Paulo, SP; Doutora em Distúrbios da Comunicação Humana.

(3) Fonoaudióloga; Diretora Clínica do CEFAC - Pós-Graduação em Saúde e Educação; Doutora em Educação pela Universidade Estadual de Campinas.

(4) Fonoaudióloga; Professora do CEFAC - Pós-Graduação em Saúde e Educação; Doutora em Ciências pela Faculdade de Medicina da Universidade de São Paulo.

(5) Fonoaudióloga; Professora Assistente Doutora da Graduação e Pós-Graduação em Fonoaudiologia da Pontifícia Universidade Católica de São Paulo, PUCSP, São Paulo,

\section{INTRODUÇÃO}

A antropometria é a ciência que estuda o corpo humano por meio de análise quantitativa, ou seja, segundo medidas de tamanho, peso e proporções ${ }^{1}$.

Trata-se de um método de análise efetivo e não invasivo $^{2}$, que pode ser direto, obtido por meio da

SP; Professora Adjunta no Curso de Fonoaudiologia da Faculdade de Ciências Médicas da Irmandade da Santa Casa de Misericórdia de São Paulo, FCM-ISCMSP, São Paulo, SP; Professora nos Cursos de Especialização em Voz da Coordenadoria Geral de Especialização, Aperfeiçoamento e Extensão da Pontifícia Universidade Católica de São Paulo, COGEAE-PUC/SP, São Paulo, SP; Professora do CEFAC - Pós-Graduação em Fonoaudiologia e Educação; Doutora em Comunicação e Semiótica pela Pontifícia Universidade Católica de São Paulo.

Conflito de interesses: inexistente 
mensuração na própria pessoa estudada, ou indireto, a partir de radiografias (cefalometria), fotografias bi ou tridimensionais ${ }^{3}$. A maneira pela qual os dados são coletados deve ser considerada ao se comparar a medida obtida e os parâmetros referidos na literatura, devido à pequena variação entre os valores adquiridos de forma direta e indireta ${ }^{4,5}$.

Estudos recentes ${ }^{5-11}$ foram realizados com 0 objetivo de fornecer dados relativos a medidas, índices e proporções antropométricas para a população brasileira. Sua importância está relacionada à escassez de pesquisas que permitem a obtenção de parâmetros de normalidade para essa população, visto que algumas medidas antropométricas utilizadas como referencial norte-americano não são compatíveis ${ }^{8,10}$. Todavia, alguns estudos $5-8,10$ dividiram a população de acordo com sexo e faixa etária. Deles, apenas dois ${ }^{6,10}$ se referiam à população adulta. Somente um estudo ${ }^{11}$ encontrado dividiu a amostra quanto ao tipo de face, mas não segundo o sexo, e foi realizado em adolescentes.

A tipologia facial é um aspecto que deveria ser considerado nos estudos que envolvem a antropometria, pois os valores das medidas podem variar dependendo do tipo de face. Existem três tipos faciais básicos: face longa ou dolicofacial, média ou mesofacial e curta ou braquifacial ${ }^{12-15}$. A tipologia facial tem relação direta com a forma de crescimento craniofacial, com a configuração das estruturas orofaciais, musculatura, funções estomatognáticas e oclusão ${ }^{12}$.

O objetivo deste trabalho foi descrever medidas antropométricas faciais de adultos, segundo tipo facial e sexo.

\section{MÉTODO}

Trata-se de um estudo descritivo e transversal, fruto de uma pesquisa qualitativa e quantitativa, direta e não invasiva. Foram selecionados 105 adultos leucodermas, 34 (32,4\%) homens e 71 $(67,6 \%)$ mulheres, com idades entre 20 e 40 anos, de uma clínica particular de ortodontia de Belo Horizonte/ MG.

Adotou-se como critérios de exclusão apresentar histórico de cirurgia ortognática e ser da raça negra ou oriental, e de inclusão possuir documentação ortodôntica com análise cefalométrica de Ricketts. Todos os pacientes realizaram a documentação em um dos dois laboratórios que prestavam serviços para a clínica e seguiam a mesma padronização para a análise cefalométrica. Não foi levado em consideração o fato dos participantes se encontrarem em tratamento ortodôntico ou o tipo de oclusão dentária apresentada.
Foi coletado da análise cefalométrica de Ricketts o valor do índice VERT baseado em cinco grandezas cefalométricas (ângulo do eixo facial, profundidade facial, ângulo do plano mandibular, altura facial inferior e do arco mandibular), o qual é utilizado para determinação do tipo de face ${ }^{16}$. Os indivíduos foram classificados em dolicofacial (índice VERT menor que -0,5), mesofacial (valores entre $-0,5$ e $+0,5$ ) e braquifacial (VERT maior que $+0,5$ ).

Em seguida, foi utilizado um protocolo baseado na literatura ${ }^{2}$ e adaptado pela pesquisadora, a qual fez uso da medição direta e não indireta, conforme proposto inicialmente pelo autor.

Foram marcados na face de cada participante com caneta de retroprojetor preta oito pontos faciais apresentados na Figura 1. São eles: násio (n)- localizado na maior depressão entre a região frontal e o nariz; mentoniano (me)- ponto mais inferior do contorno do mento; zigomático (zi)- ponto mais lateral de cada arco zigomático (foram marcados o zi direito e o zi esquerdo); subnasal (sn)- localizado na intersecção da margem inferior da base do nariz com o lábio superior; supramentoniano (b)- ponto de maior concavidade do sulco do perfil mole da mandíbula; estômio (sto)- localizado na união do lábio superior com o inferior; condílio (cd)- ponto mais superior da cabeça do côndilo da mandíbula; e gônio (go)- ponto do ângulo da mandíbula, entre a margem posterior do ramo ascendente e a base mandibular ${ }^{1}$.

Obteve-se a menor distância entre os pontos, ou seja, sete medidas lineares projetivas: altura facial anterior- násio ao mentoniano (n-me); distância bizigomática- zigomático esquerdo ao direito (zi-zi); altura do terço facial inferior- subnasal ao mentoniano (sn-me); altura da face média- násio

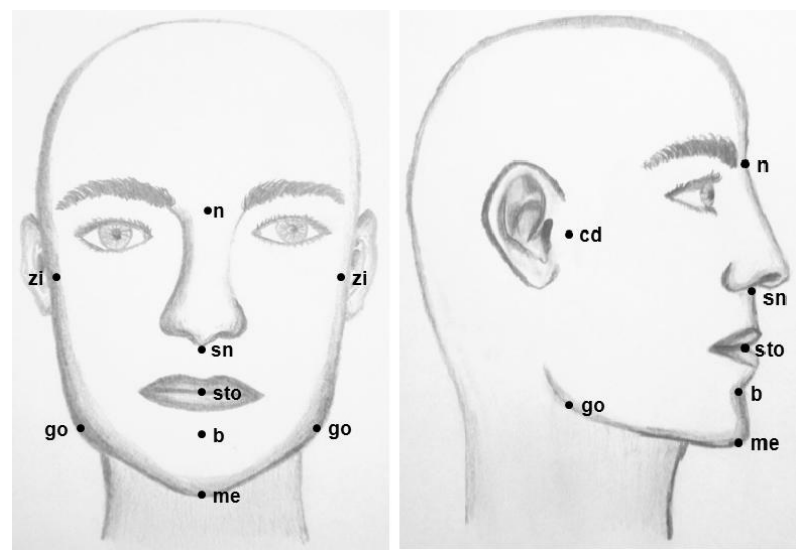

Figura 1 - Pontos antropométricos utilizados na pesquisa

Legenda: $\mathrm{n}=$ násio; $\mathrm{me}=$ mentoniano; $\mathrm{zi}=$ zigomático; sn=subnasal; b= supramentoniano; sto= estômio; $c d=$ condílio; go= gônio. 
ao estômio (n-sto); altura do queixo- supramentoniano ao mentoniano (b-me); altura da face inferior- estômio ao mentoniano (sto-me); e altura facial posterior- condílio ao gônio (cd-go).

Realizou-se as medidas por meio do uso de paquímetro de metal digital da marca Digimess 100.174BL/ Pró-fono, fabricado no Brasil, com grau de acurácia de $+/-0,02$ milímetros $(\mathrm{mm})$ e reprodutibilidade de $0,01 \mathrm{~mm}$. Para aumentar a precisão dos achados, cada medida foi mensurada três vezes durante a mesma avaliação e foi utilizada sua média aritmética. Apenas foi calculada a média entre seis valores obtidos (três medidas de cada hemi-face, esquerda e direita) para a medida da altura facial posterior (cd-go).

Para medir a distância bizigomática (zi-zi), um prolongamento de dez centímetros, projetado pela pesquisadora e confeccionado por um protético, foi adaptado ao paquímetro.

Antes de cada medição, 0 instrumento foi fechado completamente e apertado o botão de zeragem até 0 aparecimento da marcação 0,00. Ao final da coleta das medidas de cada sujeito, as hastes do paquímetro foram lavadas com água e detergente e desinfetadas por meio da fricção de algodão embebido com álcool etílico hidratado.

Os indivíduos ficaram com dentes ocluídos em posição habitual, sentados com as costas apoiadas suavemente no encosto da cadeira sem braços, pés apoiados no chão, braços soltos ao longo do corpo e sem apoio, cabeça em posição natural.
Foi solicitado que o participante olhasse para o horizonte.

Todos participantes leram e assinaram o termo de consentimento livre e esclarecido para pesquisas. Este estudo foi aprovado pelo Comitê de Ética da Pontifícia Universidade Católica de São Paulo, sob o número 0021/2006.

Os dados foram analisados de forma descritiva e comparativa, seguida de análise inferencial. Os testes estatísticos utilizados foram: teste de associação do qui-quadrado, ANOVA (análise de variância) e teste de Kruskal-Wallis, todos com nível de significância de $5 \%$. Os resultados foram cruzados e analisados segundo: sexo; tipo facial obtido por meio da cefalometria; e medidas antropométricas faciais.

\section{RESULTADOS}

Na população estudada, foram realizadas sete medições antropométricas faciais, que totalizaram uma coleta de 735 medidas.

$\mathrm{Na}$ Tabela 1, foi apresentada a descrição da amostra estudada, ou seja, o número e porcentagem dos indivíduos, segundo o tipo facial obtido por meio da análise cefalométrica e o sexo. O nível de significância obtido $(p=0,482)$ significa que não houve associação estatisticamente significativa entre sexo e tipo facial e que a distribuição dos tipos faciais foi semelhante nos dois sexos.

Tabela 1 - Número e porcentagem dos indivíduos, segundo tipo facial e sexo $(p)=0,482^{*}$

\begin{tabular}{lcccccccc}
\hline \multirow{2}{*}{ Sexo } & \multicolumn{4}{c}{ Tipo facial (cefalometria) } & \multicolumn{3}{c}{ Total } \\
\cline { 2 - 7 } & \multicolumn{2}{c}{ Dolicofacial } & \multicolumn{2}{c}{ Mesofacial } & \multicolumn{2}{c}{ Braquifacial } & & \\
\cline { 2 - 7 } & $\mathbf{N}$ & $\%$ & $\mathbf{N}$ & $\%$ & $\mathbf{N}$ & $\%$ & $\mathbf{N}$ & $\%$ \\
\hline Masculino & 7 & 25,9 & 11 & 29,7 & 16 & 39,0 & 34 & 32,4 \\
Feminino & 20 & 74,1 & 26 & 70,3 & 25 & 61,0 & 71 & 67,6 \\
\hline Total & $\mathbf{2 7}$ & $\mathbf{2 5 , 7}$ & $\mathbf{3 7}$ & $\mathbf{3 5 , 2}$ & $\mathbf{4 1}$ & $\mathbf{3 9 , 1}$ & $\mathbf{1 0 5}$ & $\mathbf{1 0 0 , 0}$ \\
\hline
\end{tabular}

Legenda: ${ }^{*}=$ nível de significância estatística segundo o teste de associação do qui-quadrado; $\mathrm{N}=$ número de sujeitos.

Os valores obtidos e calculados a partir das medições antropométricas faciais realizadas no sexo masculino e comparadas à classificação do tipo facial obtido por meio da análise cefalométrica foram expostos na Tabela 2. Os dados mostram que quatro das sete variáveis apresentaram diferenças entre as médias dos grupos.
Foram descritos na Tabela 3 os valores obtidos e calculados a partir das medições antropométricas realizadas no sexo feminino e comparadas à classificação do tipo facial obtido por meio da análise cefalométrica. Os dados retratam que cinco das sete variáveis apresentaram diferenças entre as médias dos grupos. 
Tabela 2 - Medidas antropométricas faciais, segundo os tipos faciais, no sexo masculino

\begin{tabular}{|c|c|c|c|c|c|}
\hline Variável & Tipo facial & $\mathbf{N}$ & Média (em mm) & Desvio-padrão & $\mathbf{p}$ \\
\hline \multirow{3}{*}{$n-m e^{A}$} & Dolicofacial & 7 & 128,45 & 4,04 & \multirow{3}{*}{$<0,001^{x}$} \\
\hline & Mesofacial & 11 & 121,79 & 6,78 & \\
\hline & Braquifacial & 16 & 115,91 & 5,93 & \\
\hline \multirow{3}{*}{$z i-z i^{A}$} & Dolicofacial & 7 & 118,02 & 5,60 & \multirow{3}{*}{0,324} \\
\hline & Mesofacial & 11 & 121,26 & 6,94 & \\
\hline & Braquifacial & 16 & 117,89 & 5,32 & \\
\hline \multirow{3}{*}{ sn-me ${ }^{A}$} & Dolicofacial & 7 & 75,05 & 4,53 & \multirow{3}{*}{$0,002^{*}$} \\
\hline & Mesofacial & 11 & 70,01 & 4,64 & \\
\hline & Braquifacial & 16 & 66,67 & 4,76 & \\
\hline \multirow{3}{*}{ n-sto ${ }^{A}$} & Dolicofacial & 7 & 79,03 & 3,44 & \multirow{3}{*}{$<0,001^{*}$} \\
\hline & Mesofacial & 11 & 75,42 & 3,56 & \\
\hline & Braquifacial & 16 & 70,70 & 3,79 & \\
\hline \multirow{3}{*}{$b-m e^{A}$} & Dolicofacial & 7 & 31,23 & 3,89 & \multirow{3}{*}{0,265} \\
\hline & Mesofacial & 11 & 28,66 & 4,13 & \\
\hline & Braquifacial & 16 & 28,59 & 3,28 & \\
\hline \multirow{3}{*}{ sto-me ${ }^{A}$} & Dolicofacial & 7 & 53,08 & 3,79 & \multirow{3}{*}{$0,006^{*}$} \\
\hline & Mesofacial & 11 & 48,39 & 4,39 & \\
\hline & Braquifacial & 16 & 46,42 & 4,27 & \\
\hline \multirow{3}{*}{$\operatorname{cd}-g o^{A}$} & Dolicofacial & 7 & 51,99 & 3,18 & \multirow{3}{*}{0,387} \\
\hline & Mesofacial & 11 & 54,68 & 6,62 & \\
\hline & Braquifacial & 16 & 54,98 & 3,98 & \\
\hline
\end{tabular}

Legenda: ${ }^{A}=$ nível de significância estatística segundo a ANOVA (análise de variância); ${ }^{*}=$ resultados estatisticamente significativos; $\mathrm{N}=$ número de sujeitos; $\mathrm{n}-\mathrm{me}=$ altura facial anterior (násio ao mentoniano); zi-zi= distância bizigomática (zigomático esquerdo ao direito); sn-me= altura do terço facial inferior (subnasal ao mentoniano); n-sto= altura da face média (násio ao estômio); b-me= altura do queixo (supramentoniano ao mentoniano); sto-me= altura da face inferior (estômio ao mentoniano); $c \mathrm{~d}=\mathrm{go}=$ altura facial posterior (condílio ao gônio).

\section{DISCUSSÃO}

Atualmente, a antropometria é utilizada para diagnóstico e acompanhamento de diversos tratamentos médicos e de áreas afins ${ }^{17}$. A utilização de algumas medidas antropométricas faciais é indicada durante a avaliação realizada pelo fonoaudiólogo que atua em motricidade orofacial ${ }^{5,18}$.

A pesquisa foi efetuada com a população de uma clínica de ortodontia com a possibilidade de acesso à análise cefalométrica, utilizada em todos os pacientes. Deve-se ressaltar que foi dada atenção especial à forma de análise feita pelos laboratórios para se averiguar que seguiam a mesma padronização.

Participaram apenas indivíduos leucodermas, assim como em vários estudos ${ }^{7,8,10,11,19-21}$. A preocupação se deu por causa da variação das raças com relação às estruturas craniofaciais ${ }^{6,22,23}$.

Para a coleta das medidas antropométricas faciais, todas as etapas metodológicas foram consideradas, conforme preconizado: localização precisa e palpação antes da marcação dos pontos antropométricos; utilização do instrumento paquímetro para mensuração de distâncias lineares projetivas entre dois pontos do tecido mole, sem pressionar a superfície da pele; examinador em posição sentada à frente do pesquisado; sujeito avaliado sentado com cabeça em posição natural 1,19,24. Além disso, foi orientado que os dentes permanecessem em oclusão na posição habitual, ou seja, sem máxima intercuspidação ${ }^{19}$. Como ocorreu em outros trabaIhos 17,22,23, todas as medições foram coletadas pela mesma pesquisadora para que não houvesse variação entre diferentes avaliadores.

Com relação aos resultados, foi encontrado maior número de pessoas do sexo feminino diante do masculino na amostra estudada (Tabela 1), da mesma forma que ocorreu em outras pesquisas 20,25,26 que também estudaram a população de clínicas de ortodontia. A maior freqüência do sexo feminino na população estudada, ou seja, de uma clínica ortodôntica, pode estar relacionada às mulheres serem mais atentas com estética e saúde. 
Tabela 3 - Medidas antropométricas faciais, segundo os tipos faciais, no sexo feminino

\begin{tabular}{|c|c|c|c|c|c|}
\hline Variável & Tipo facial & $\mathbf{N}$ & Média (em mm) & Desvio-padrão & $p$ \\
\hline \multirow{3}{*}{$n-m e^{A}$} & Dolicofacial & 20 & 115,24 & 6,38 & \multirow{3}{*}{$0,006^{*}$} \\
\hline & Mesofacial & 26 & 115,07 & 4,92 & \\
\hline & Braquifacial & 25 & 110,58 & 5,42 & \\
\hline \multirow{3}{*}{$z i-z i^{A}$} & Dolicofacial & 20 & 114,37 & 6,40 & \multirow{3}{*}{0,613} \\
\hline & Mesofacial & 26 & 114,48 & 4,69 & \\
\hline & Braquifacial & 25 & 113,05 & 5,81 & \\
\hline \multirow{3}{*}{ sn-me ${ }^{A}$} & Dolicofacial & 20 & 65,94 & 5,47 & \multirow{3}{*}{$0,001^{*}$} \\
\hline & Mesofacial & 26 & 65,29 & 3,59 & \\
\hline & Braquifacial & 25 & 61,18 & 4,10 & \\
\hline \multirow{3}{*}{ n-sto ${ }^{A}$} & Dolicofacial & 20 & 72,78 & 4,40 & \multirow{3}{*}{$0,005^{\star}$} \\
\hline & Mesofacial & 26 & 71,59 & 3,90 & \\
\hline & Braquifacial & 25 & 68,89 & 3,84 & \\
\hline \multirow{3}{*}{$b-m e^{A}$} & Dolicofacial & 20 & 26,15 & 2,68 & \multirow{3}{*}{0,332} \\
\hline & Mesofacial & 26 & 26,55 & 1,99 & \\
\hline & Braquifacial & 25 & 25,49 & 2,90 & \\
\hline \multirow{3}{*}{ sto-me ${ }^{B}$} & Dolicofacial & 20 & 45,12 & 4,35 & \multirow{3}{*}{$0,026^{*}$} \\
\hline & Mesofacial & 26 & 44,84 & 2,66 & \\
\hline & Braquifacial & 25 & 42,66 & 2,66 & \\
\hline \multirow{3}{*}{$\mathrm{cd}_{-} \mathrm{go}^{\mathrm{A}}$} & Dolicofacial & 20 & 45,89 & 4,10 & \multirow{3}{*}{$0,001^{*}$} \\
\hline & Mesofacial & 26 & 49,39 & 3,77 & \\
\hline & Braquifacial & 25 & 50,38 & 3,69 & \\
\hline
\end{tabular}

Legenda: ${ }^{A}=$ nível de significância estatística segundo a ANOVA (análise de variância); ${ }^{B}=$ nível de significância estatística segundo 0 teste Kruskal-Wallis; * = resultados estatisticamente significativos; $\mathrm{N}=$ número de sujeitos; $\mathrm{n}$-me= altura facial anterior (násio ao mentoniano); zi-zi= distância bizigomática (zigomático esquerdo ao direito); sn-me= altura do terço facial inferior (subnasal ao mentoniano); $\mathrm{n}$-sto= altura da face média (násio ao estômio); b-me= altura do queixo (supramentoniano ao mentoniano); sto-me= altura da face inferior (estômio ao mentoniano); $\mathrm{cd}=\mathrm{go}=$ altura facial posterior (condílio ao gônio).

A partir do estudo comparativo das Tabelas 2 e 3, foram observados, no geral, valores médios maiores para os homens quando comparados às mulheres, como descrito na literatura ${ }^{17,19,27}$.

Com relação à medida da altura facial anterior (násio- $\mathrm{n}$ ao mentoniano-me), as médias apresentaram diferenças estatisticamente significantes para ambos os sexos. Os valores seguiram a ordem decrescente de dolicofacial>mesofacial> braquifacial, tanto para homens como mulheres. Esse resultado era esperado, pois, segundo a literatura ${ }^{12,28}$, a face longa apresenta o maior valor dessa medida quando comparada aos outros tipos faciais e a face curta o menor. Valores maiores foram encontrados em outro estudo, no qual essa medida foi colhida de forma indireta a partir de traçados realizados em fotografias de indivíduos norte-americanos ${ }^{19}$. Pode-se atribuir a essa divergência dos achados o fato da metodologia usada ser diferente, seja pela forma de coleta ou pela raça diferentes.

No que diz respeito à medida da distancia bizigomática (zigomático esquerdo ao direito- zi-zi), as médias não apresentaram diferenças estatisticamente significantes em nenhum dos sexos. Os valores para ambos os sexos seguiram a ordem mesofacial>dolicofacial>braquifacial, o que não condiz com o fato da face longa ser caracterizada como mais estreita e a face curta mais larga ${ }^{12,17,28}$. Entretanto, ao ser realizado um estudo com diferentes raças e etnias, os autores apontaram que essa foi a medida com a qual se obteve resultados mais divergentes ${ }^{21}$. Outro estudo mostrou que os indivíduos de origem africana apresentam medidas faciais, principalmente as horizontais, maiores que as dos indivíduos caucasianos ${ }^{23}$. Resultado diferente foi obtido em outra pesquisa na qual quase não foram encontradas diferenças dessa medida ao se comparar indivíduos afro-americanos e caucasianos em ambos os sexos ${ }^{22}$. Pode-se acrescentar que, ao se comparar com esta pesquisa, valores médios maiores para ambos os sexos foram encontrados nos resultados de alguns estudos ${ }^{21-23} \mathrm{e}$ menores em outro ${ }^{17}$. Nesses casos, os estudos tiveram como público-alvo raças e etnias diferentes da utilizada neste estudo. Esse achado pode ter 
relação com o fato de existir variação das medidas entre a população leucoderma brasileira e as outras raças e etnias apresentadas nos estudos, ou pode estar relacionada à grande miscigenação racial da população brasileira. Outra razão pode ser referente ao uso da adaptação no paquímetro para coleta dessa medida, mas adaptação semelhante foi feita em outros estudos que não relataram incongruência de achados ${ }^{29,30}$.

Para a medida da altura do terço facial inferior (subnasal-sn ao mentoniano-me), foram encontradas diferenças estatisticamente significantes tanto nos homens como nas mulheres. Os valores médios encontrados, para ambos os sexos, seguiram a seguinte ordem: dolicofacial>mesofaci al>braquifacial. Esse achado era esperado, pois 0 terço inferior do indivíduo de face longa é considerado mais longo e na face curta, diminuído diante dos demais terços ${ }^{12,28}$. Em um estudo em que se coletou essa medida indiretamente a partir de fotografias, os valores médios foram maiores que os desta pesquisa, tanto para os homens como para as mulheres ${ }^{19}$.

Com relação à medida da altura da face média (násio-n ao estômio-sto), as médias encontradas apresentaram diferenças estatisticamente significantes em ambos os sexos. Os valores das médias seguiram a ordem de dolicofacial>mesofacial>braq uifacial, para homens e mulheres. Esse dado coincide com o que é apontado na literatura ${ }^{12,28}$, ou seja, terço facial médio mais longo para o indivíduo dolicofacial e curto, para o braquifacial. Os valores encontrados nesta pesquisa foram menores, nos dois sexos, daqueles apontados por um estudo no qual se realizou medição direta de indivíduos caucasianos adultos ${ }^{27}$ e maiores dos apresentados em outra pesquisa na qual o público alvo foram turcos adultos ${ }^{17}$

Quanto à medida da altura do queixo (supramentoniano-b ao mentoniano-me), as médias obtidas não apresentaram diferenças estatisticamente significantes em nenhum dos sexos. As ordens decrescentes diferentes encontradas ao se comparar os valores médios foram: dolicofacial $>$ me sofacial>braquifacial, para homens; e mesofacial $>d$ olicofacial>braquifacial, para mulheres. Não foram encontrados na literatura pesquisada valores dessa medida para comparação.

No que diz respeito à altura da face inferior (estômio-sto ao mentoniano-me), as médias obtidas apresentaram diferenças estatisticamente significantes em ambos os sexos. Os valores seguiram a ordem de dolicofacial>mesofacial $>$ braq uifacial. Esse achado está de acordo com o que é referido na literatura ao se caracterizar a face longa por excesso vertical ósseo maxilar e mandibular na região anterior, e a face curta por dimensão vertical intra-oral diminuída e altura facial anterior reduzida ${ }^{12,28}$. Os valores encontrados na pesquisa foram relativamente pouco menores que os verificados em um estudo em que essa medida foi adquirida indiretamente a partir de fotografias ${ }^{19}$.

A última medida a ser discutida é a altura facial posterior (condílio-cd ao gônio-go). As médias apenas apresentaram diferenças estatisticamente significantes no sexo feminino. Os valores, em ambos os sexos, seguiram a seguinte ordem: braq uifacial>mesofacial>dolicofacial. Esse dado condiz com a literatura: face longa caracterizada por ramo mandibular estreito e curto; média por mandíbula com ramo e corpo totalmente desenvolvidos com largura do ramo ascendente igual à altura do corpo; e curta por mandíbula com ramo largo e longo ${ }^{12,28}$. Não foram encontrados estudos que apresentassem valores relativos a essa medida para comparação.

A partir da realização desta pesquisa, algumas sugestões para estudos subseqüentes podem ser apresentadas: coletar esses dados em outras amostras e as pesquisas que caracterizam a face por meio da antropometria poderiam se preocupar em separar sua população não apenas segundo sexo, idade e raça, mas também de acordo com tipos faciais. Isso porque os dados parecem indicar que uma maior ou menor porcentagem de determinado tipo facial, em um dado grupo analisado, podem influenciar os resultados obtidos das médias gerais.

\section{CONCLUSÃO}

Em vista dos resultados expostos e discutidos, pode-se concluir que:

1. Houve dimorfismo sexual para todas as medidas antropométricas obtidas, as quais tiveram valores médios maiores para o sexo masculino quando comparados ao feminino;

2. Os tipos faciais classificados por meio da cefalometria apresentaram diferenças estatisticamente significantes para os valores médios das seguintes medidas antropométricas faciais: altura facial anterior ( $\mathrm{n}$-me), altura do terço facial inferior (sn-me), altura da face média (n-sto) e altura da face inferior (sto-me) para o sexo masculino; e altura facial anterior (n-me), altura do terço facial inferior (sn-me), altura da face média (n-sto), altura da face inferior (sto-me) e altura facial posterior (cd-go) para o feminino.

\section{AGRADECIMENTOS}

À CAPES, pelo auxílio financeiro e pelo incentivo à pesquisa. 


\section{ABSTRACT}

Purpose: to describe anthropometric measurements in adults according to facial type and gender. Method: 105 Caucasian adults took part, 34 male (32.4\%) and 71 female (67.6\%) with ages between 20 and 40-year old, in a private orthodontic clinic in Belo Horizonte, Minas Gerais, Brazil. The findings from the cephalometric analysis were compared for determining facial type with seven direct anthropometric measurements: anterior face height- nasion to menton; distance bizygomatiquezygion left to right; lower face height- subnasale to menton; middle face height- nasion to stomion; chin height- supramenton to menton; inferior face height- stomion to menton; and posterior face heightcondylion to gonion. Results: for male, the facial types classified by means of cephalometry had significant statistical differences for the average values of the anthropometric measurements: anterior face height, lower face height, middle face height and inferior face height. For female, significant statistical differences were found in the following measurements: anterior face height, lower face height, middle face height, inferior face height and posterior face height. Conclusion: there was sexual dimorphism for all collected anthropometric measurements. The male showed higher average values when compared with the female. The facial types classified by means of cephalometry showed significant statistical differences in four anthropometric measurements, for male and five for female.

KEYWORDS: Face; Measures; Anthropometry; Adult

\section{REFERÊNCIAS}

1. Farkas LG. Anthropometry of the head and face. 2a ed. New York: Raven Press; 1994. p.1-77.

2. Jacobson RL. Facial analysis in two and three dimensions. In: Jacobson A. Radiographic cephalometry- from basics to videoimaging. Chicago: Quintessence Publishing Co; 1995. p.273-94.

3. Weinberg SM, Naidoo S, Govier DP, Martin RA, Kane AA, Marazita ML. Anthropometric precision and accuracy of digital three-dimensional photogrammetry: comparing the genex and 3dMD imaging systems with one another and with direct anthropometry. J Craniofac Surg 2006;17(3):477-83.

4. Farkas LG, Tompson BD, Katic MJ, Forrest CR. Differences between direct (anthropometric) and indirect (cephalometric) measurements of the skull. J Craniofac Surg 2002;13(1):105-8.

5. Cattoni DM. O uso do paquímetro na avaliação da morfologia orofacial. Rev Soc Bras Fonoaudiol 2006;11(1):52-8.

6. Parro FM, Toledo MR, Gomes ICD, Marchesan IQ. Diferenças antropométricas entre mulheres brancas e negras após crescimento puberal. Rev CEFAC 2005;(4):459-65.

7. Cattoni DM, Fernandes FDM, Di Francesco RC, Latorre MRDO. Características do sistema estomatognático de crianças respiradoras orais: enfoque antroposcópico. $\mathrm{R}$ Pró-fono 2007;19(4):347-51.
8. Cattoni DM, Fernandes FDM. Medidas antropométricas orofaciais de crianças paulistanas e norte-americanas: estudo comparativo. R Pró-fono 2009;21(1):25-30.

9. Cunha DA, Renata MFL, Nascimento GKBO, Silva EGF, Silva HJ, Prado IJ, et al. Antropometria e mastigação em crianças asmáticas. Rev CEFAC 2009;11(Supl3):341-8.

10. Sant'Ana E, Kuriki EU, Arnett W, Lautenschläger GAC, Yaedu RYF. Avaliação comparativa do padrão de normalidade do perfil facial em pacientes brasileiros leucodermas e em norte-americanos. $R$ Dental Press Ortodon Ortop Facial. 2009; 14(1):80-9. 11. Guedes SPC, Teixeira BV, Cattoni DM. Medidas orofaciais em adolescentes do estado do Rio de Janeiro segundo a tipologia facial. Rev CEFAC 2010;12(1):68-74.

12. Bianchini EMG. A cefalometria nas alterações miofuncionais orais - diagnóstico e tratamento fonoaudiológico. 5a ed rev. e ampl. Carapicuíba: Pró-fono; 2002.

13. Ramires RR, Ferreira LP, Marchesan IQ, Cattoni DM, Andrada e Silva MA. Relação entre cefalometria e análise facial na determinação do tipo de face. Rev CEFAC 2009;11(Supl3):349-54.

14. Vianna-Lara MS, Caria PHF, Tosello DO, Lara F, Amorim MM. Electromyographic activity of masseter and temporal muscles with different facial types. Angle Orthod 2009;79(3):515-20.

15. Ramires RR, Ferreira LP, Marchesan IQ, Cattoni DM, Andrada e Silva MA. Tipologia facial aplicada à Fonoaudiologia: revisão de literatura. Rev Soc Bras Fonoaudiol 2010;15(1):140-5. 
16. Rickets RM. Orthodontic diagnosis and planning- their roles in preventive and rehabilitative dentristy. Denver: Rocky Mountain Orthodontics; 1982. 269p.

17. Arslan SG, Genç C, Odabaş B, Kama JD. Comparison of facial proportions and anthropometric norms among Turkish young adults with different face types. Aesth Plast Surg 2008;32:234-42.

18. Rodrigues FV, Monção FRC, Moreira MBR, Motta AR. Variabilidade na mensuração das medidas orofaciais. Rev Soc Bras Fonoaudiol 2008;13(4):332-7.

19. Arnett GW, Jelic JS, Kim J, Cummings DR, Beress A, Worley Jr CM, Chung B, Bergman R. Soft tissue cephalometric analysis: diagnosis and treatment planning of dentofacial deformity. Am J Orthod Dentofacial Orthop 1999;116(3):239-53.

20. Daenecke S, Bianchini EMG, Silva APBV. Medidas antropométricas de comprimento de lábio superior e filtro. R Pró-fono 2006;18(3):249-58.

21. Farkas LG, Katic MJ, Forrest CR. International anthropometric study of facial morphology in various ethnic groups/ races. J Craniofac Surg 2005;16(4):615-46.

22. Farkas LG, Katic MJ, Forrest CR. Comparison of craniofacial measurements of young adult africanamerican and north american white males and females. Ann Plast Surg 2007;59(6):692-8.

23. Porter JP, Olson KL. Anthropometric facial analysis of the african american woman. Arch Facial Plast Surg 2001;3(3):191-7.
24. Feres R, Vasconcelos MHF. Estudo comparativo entre a análise facial subjetiva e a análise cefalométrica de tecidos moles no diagnóstico ortodôntico. R Dental Press Ortodon Ortop Facial 2009;14(2):81-8.

25. Canuto MSB, Assis RS, Gouveia PMP, Nemr K. Análise comparativa entre presença de diastemas e tipos faciais. Rev CEFAC 2006;8(2):162-70.

26. Pieri LV, Faltin Júnior K, Ortolani CLF, Faltin RM, Almeida MAA. Crescimento da base craniana nos diferentes tipos faciais nos relacionamento maxilomandibulares ortopédicos de classe I, II e III - Parte 1. R Dental Press Ortodon Ortop Facial 2007;12(1):110-7.

27. Budai M, Farkas LG, Tompson B, Katic M, Forrest CR. Relation between anthropometric measurements and proportions of the face of healthy young white adult men and women. J Craniofac Surg 2003;14(3)154-63.

28. Enlow DH, Poston WR, Bakor SF. Crescimento facial. 3a ed. Artes Médicas; 1993.

29. Bianchini AP, Guedes ZCF, Vieira MM. Estudo da relação entre a respiração oral e o tipo facial. Rev Bras Otorrinolaringol 2007;73(4):500-5.

30. Sies ML, Farias SR, Vieira MM. Respiração oral: relação entre o tipo facial e a oclusão dentária em adolescentes. Rev Soc Bras Fonoaudiol 2007;12(3):191-8.

DOI: 10.1590/S1516-18462010005000128

RECEBIDO EM: 01/06/2010

ACEITO EM: 09/08/2010

Endereço para correspondência:

Rossana Ribeiro Ramires

Rua Teopompo de Vasconcelos, 375/ 163

Vila Adyana

São José dos Campos/SP

CEP: 12243-830

E-mail: rossana_ramires@yahoo.com.br 